\title{
Calcitriol Reduces Eosinophil Necrosis Which Leads to the Diminished Release of Cytotoxic Granules
}

\author{
Caroline Ethier $^{\mathrm{a}}$ Yingqi Yu $^{\mathrm{a}}$ Lisa Cameron $^{\mathrm{c}}$ Paige Lacy $^{\mathrm{a}}$ Francis Davoine $^{\mathrm{a}, \mathrm{b}}$

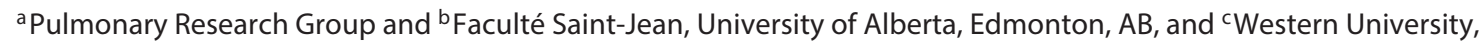 \\ London, ON, Canada
}

\section{Keywords}

Eosinophils · Apoptosis · Inflammation · Cytotoxicity

\begin{abstract}
Background: Asthma severity and eosinophilia correlate with a deficiency in vitamin $\mathrm{D}$ and its active metabolite calcitriol. Calcitriol modulates numerous leukocyte functions, but its effect on eosinophils is not fully understood. We postulated that calcitriol exerts a direct effect on eosinophil biology by modulating cell survival. Methods: Purified peripheral blood eosinophils from atopic donors were incubated in the presence of calcitriol for up to 14 days with or without IL-5. The effect of calcitriol on eosinophil viability was measured using the annexin-V/propidium iodide flow cytometry assay. We also examined the release of eosinophil peroxidase (EPX) in media using a flow cytometry assay with antiEPX antibodies, and the enzymatic activity of EPX was measured by an OPD-based colorimetric assay. Results: We observed that calcitriol sustained cell viability in eosinophils with a concurrent reduction of necrotic cells. This effect was amplified by the addition of IL-5. In parallel, we observed that a physiological dose of calcitriol ( $10 \mathrm{nM})$ significantly reduced eosinophil necrosis and cytolytic release of EPX in media when coincubated with IL-5. Conclusion: These results suggest that calcitriol may exert a direct effect on eosinophils by reducing necrosis and the cytolytic release of inflammatory mediators like EPX.

(c) 2016 S. Karger AG, Basel
\end{abstract}

(C) 2016 S. Karger AG, Basel

E-Mail karger@karger.com

www.karger.com/iaa

\section{Introduction}

The eosinophilic granulocyte is one of the most prominent inflammatory cells characterizing allergic airway inflammation. Lower numbers of eosinophils in lung tissues and the blood broadly correlate with an improvement in allergic symptoms, and have been generally considered as a sign of improved control of bronchial inflammation [1]. Eosinophils are known for their capacity to release (i.e., degranulate) highly cytotoxic granules and also a variety of mediators, which can cause direct tissue injury and incite inflammation [2-5]. One major mode of eosinophil degranulation is cytolysis $[6,7]$. During cytolysis, also referred to as cytolytic degranulation, mediator release occurs spontaneously or is linked to necrosis. Fairly recent work has reported an association between the regulation of eosinophil death and the pathological role of eosinophils in the progression of allergic inflammation $[8,9]$. More specifically, it is now accepted that delayed or insufficient apoptosis of eosinophils is an important factor in the pathology of asthma [10-12]. In fact, airway inflammation caused by asthma-associated insults, such as epithelial shedding and allergic immune responses, induces the rapid necrotic death of eosinophils, causing granule deposition throughout the airway tissues [7]. Cytolysis, causing a release of intracellular components, is the characteristic result of necrotic death. Necrosis in eosinophils seems to be a significant proin- 
flammatory process in disease, since eosinophil cytolysis has often been observed in human $[6,13-16]$ and animal [17] tissues undergoing eosinophilic inflammation. Therefore, we developed an interest in modulating eosinophil cell death to avoid eosinophilic cytotoxic mediator release with the goal of minimizing the presence of the proinflammatory components originating from these granulocytes within their surrounding environments.

One candidate that may influence eosinophil survival and the cytolytic release of eosinophil granules is the vitamin $\mathrm{D}_{3}$ active metabolite, calcitriol. There is extensive literature about the ability of calcitriol to evoke cell cycle arrest and increase apoptotic cancer cells $[18,19]$. Moreover, a report showed that 1,25-dihydroxyvitamin $\mathrm{D}_{3}$ was able to maintain the survival of peripheral blood eosinophils from healthy donors, although the effects of calcitriol on cytolytic degranulation in eosinophils was not characterized [20].

Vitamin D is a fat-soluble lipid within the secosteroid group, primarily known to play an essential role in the regulation of calcium absorption from the gastrointestinal tract. There is considerable evidence supporting alternative functions of vitamin $\mathrm{D}$ beyond calcium regulation. Despite the well-known role of calcitriol in immune responses, the mechanism by which calcitriol may mitigate eosinophil function is largely unknown. The major aim of this study was to understand whether it is possible to regulate eosinophil survival and cytolytic degranulation in eosinophils.

In this study, we report that calcitriol can directly regulate eosinophil inflammatory effects by reducing the necrotic release of intact granules as well as active eosinophil peroxidase (EPX). We propose that reducing eosinophil cytolytic degranulation of proinflammatory mediators could minimize mucosal tissue injury in airway allergic inflammatory pathologies.

\section{Materials and Methods}

\section{Blood Donors}

Adult blood donors were recruited from the general public in the area of Edmonton, AB. Blood collection was restricted to adult donors (i.e., at least 18 years old) who had mild eosinophilia (i.e., $>4 \%$ of total white blood cell count) as well as self-reported allergies and/or allergic asthma, but not currently using corticosteroids. Approval for the study was obtained from the local Ethics Research Board at the Faculty of Medicine and Dentistry (University of Alberta) and all adult subjects gave their informed consent according to the Helsinki protocol.

\section{Peripheral Blood Eosinophil Isolation}

Peripheral blood eosinophils from atopic donors were purified as previously described [21]. Briefly, venous blood (100 mL) was collected from the cephalic or median cubital veins in EDTA-coated vacutainers (BD Vacutainer ${ }^{\circledR}$, BD Hemogard ${ }^{\mathrm{TM}}$, Franklin Lakes, NJ, USA). Erythrocytes were sedimented using a dextran gradient (6\% in RPMI) (Sigma-Aldrich Canada Ltd., Oakville, ON, Canada). Granulocytes were separated from mononuclear cells by centrifugation using Ficoll-Paque ${ }^{\mathrm{TM}}$ Plus (GE-HealthCare Ltd., Uppsala, Sweden). Eosinophils were further purified using EasySep human eosinophil enrichment kit (STEMCELL Technologies ${ }^{\mathrm{TM}}$, Vancouver, BC, Canada) as per manufacturer's instructions. Purity of eosinophil preparations were $>98 \%$ (contaminating cells were neutrophils and/or lymphocytes).

Viability Assay

Viability levels were measured using the Alexa Fluor ${ }^{\circledR} 488$ Annexin V/Dead Cell apoptosis kit (Invitrogen ${ }^{\mathrm{TM}}$, Life Technologies, Eugene, OR, USA) according to the manufacturer's instructions. Acquisition was performed using a BD FACSCanto flow cytometer. Viable cells were double-negative, while necrotic cells were double-positive for annexin-V and propidium iodide (PI).

\section{EPX and Eosinophil Granule Staining}

Paraformaldehyde-fixed eosinophil supernatants were labeled with anti-CD63 (AbD Serotec, Kensington, Oxford, England) or matched isotype control (AbD Serotec) antibodies, in order to detect granules. These eosinophils were costained with anti-EPX-biotin (a gift from Dr. Lee, Mayo Clinic, AZ, USA) and streptavidin (Affymetrix eBioscience Inc., San Diego, CA, USA). Both detections were done using the manufacturer's protocols. The levels of free EPX and intact granules were obtained by BD FACSCanto flow cytometer analysis.

\section{EPX Colorimetric Assay}

The measurement of EPX release has been described [21]. Briefly, eosinophil suspensions in phenol red-free RPMI (SigmaAldrich) were distributed in a 96-well plate to contain approximately 30,000 cells per well. For the lysed control, eosinophils were subject to $-80^{\circ} \mathrm{C}$ freezing and mechanical stress. Platelet-activating factor $(\mathrm{PAF})_{\mathrm{C} 16}$ (Sigma-Aldrich, St. Louis, $\mathrm{MO}$, USA) was added to the respective wells for $5 \mathrm{~min}$ prior to the addition of the peroxidase substrate solution (OPD $0.9 \mathrm{mg} / \mathrm{mL}, \mathrm{CaCl}_{2} 1.6 \mathrm{~mm}$, $0.004 \% \mathrm{H}_{2} \mathrm{O}_{2}$ in HBSS) $1 \times$ as a positive control. The plate was incubated for $2 \mathrm{~min}$ before the reaction was stopped using $4 \mathrm{M} \mathrm{H}_{2} \mathrm{SO}_{4}$ (Sigma-Aldrich Canada Ltd.). Results were calculated as:

$\% E P X$ released into media $=\frac{E P X \text { activity in media }(\text { with intact cells })}{E P X \text { activity in media of cell lysate }} \times 100 \%$.

Each experiment was done in triplicate and absorbance at 450 $\mathrm{nm}$ wavelength was read using a BioTek ${ }^{\circledR}$ PowerWave XS plate reader. All values were normalized to blank readings.

\section{Statistical Analysis}

All results are expressed as mean \pm standard error of the mean. Comparison between the groups was done using one-way ANOVA and paired $t$ test statistical tests (GraphPad Prism ${ }^{\circledR}$ software) where a $p$ value $<0.05$ was considered significant. 

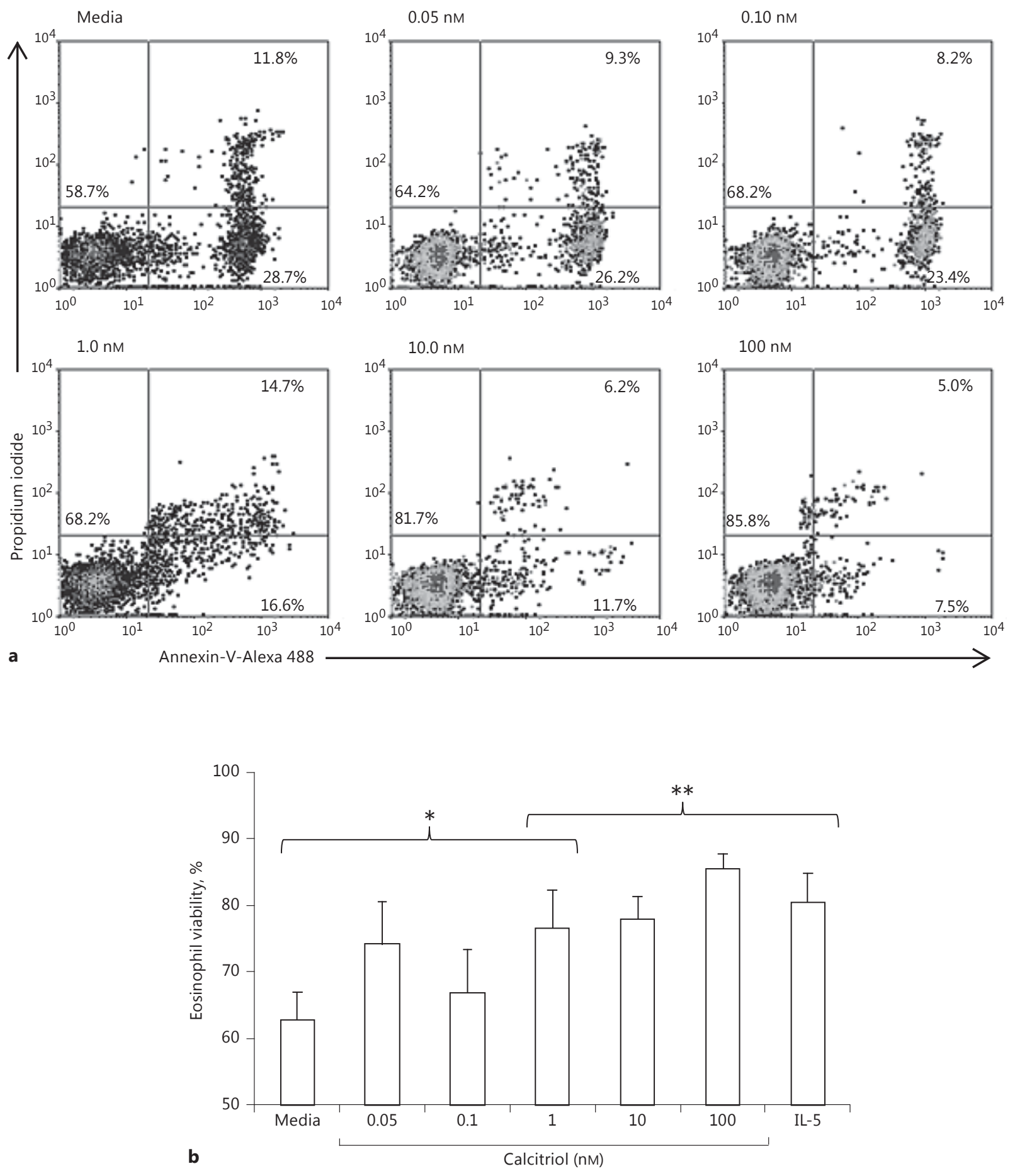

Fig. 1. Increasing concentrations of calcitriol $\left(1 \alpha, 25-[\mathrm{OH}]_{2} \mathrm{D}_{3}\right) \mathrm{im}$ prove the survival of human blood-derived eosinophils ex vivo over a 24-h period. a The number of double-positive eosinophils for annexin- $V$ and propidium iodide (i.e., the necrotic cell population in the right upper quadrant) decreases while the number of double-negative eosinophils increases according to calcitriol concentration augmentation. $\mathbf{b}$ The percentage of viable eosinophils is improved after $24 \mathrm{~h}$ of treatment with calcitriol (0.05-1 nM) in a dose-dependent manner; eosinophil survival is significantly lower than with IL-5 treatment $(1 \mathrm{ng} / \mathrm{mL})(n=5, * p<0.05)$. Viability with calcitriol $(1-100 \mathrm{nM})$ is significantly higher than with media ( $\left.n=5,{ }^{* *} p<0.05\right)$. Viability was determined by annexin- $\mathrm{V}$ binding and propidium iodide exclusion on flow cytometry. 


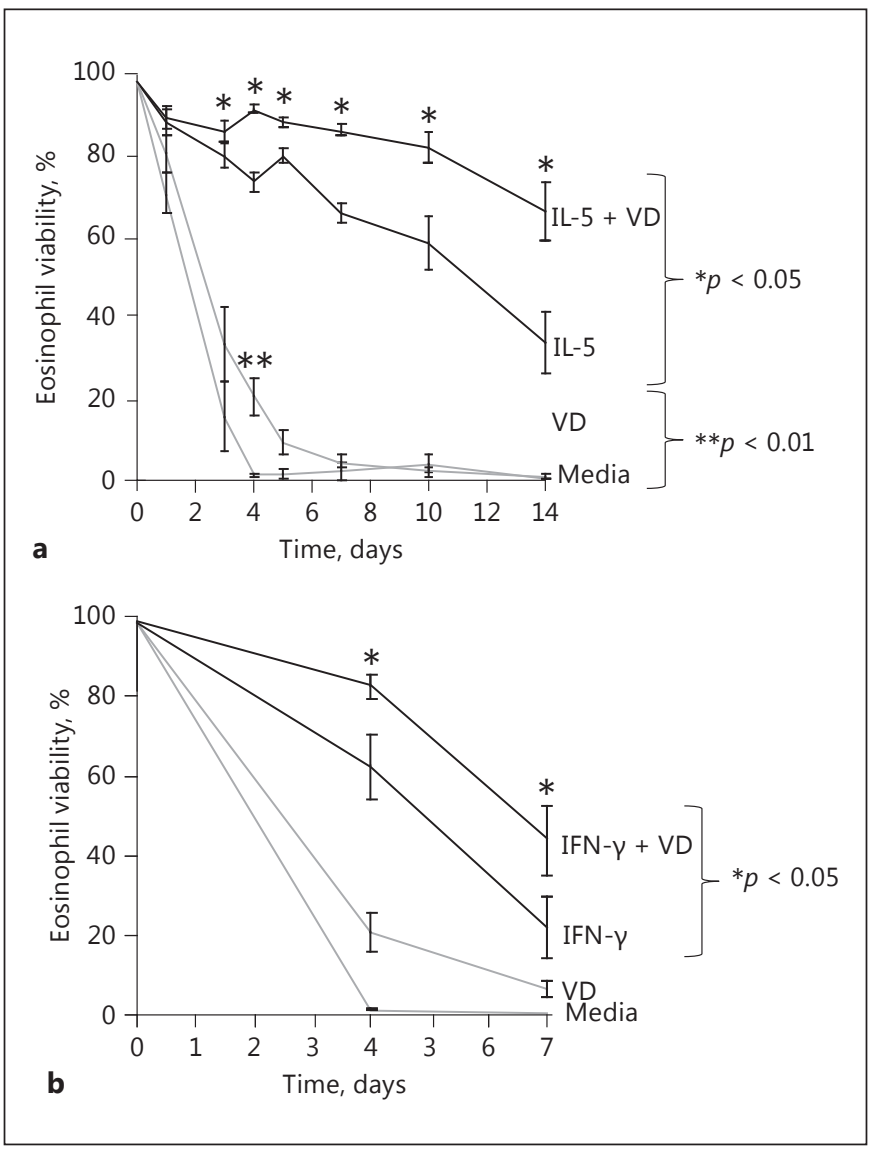

Fig. 2. Calcitriol potentiates the survival effect of the antiapoptotic cytokines, IL- 5 and IFN- $\gamma$, on human peripheral blood eosinophils ex vivo. a IL-5 (1 ng/mL) plus calcitriol $(10 \mathrm{nM})$ had an additive effect from day 4 , and synergistically prolonged eosinophil viability as of day 7 of incubation (IL-5 + VD versus IL-5; ${ }^{*} p<0.05, n=$ 12). b Calcitriol (10 nM) in combination with IFN- $\gamma(100 \mathrm{ng} / \mathrm{mL})$ also had a synergistic effect on eosinophil survival at day 7 of incubation (IFN- $\gamma+$ VD vs. IFN- $\gamma$; $\left.{ }^{*} p<0.05, n=5\right)$. VD, vita$\min \mathrm{D}$.

\section{Results}

\section{Calcitriol Modulates Eosinophil Survival}

To determine if vitamin $\mathrm{D}$ influences eosinophil survival, we investigated whether the most active metabolite of vitamin $\mathrm{D}_{3}$, calcitriol $\left(1 \alpha, 25-[\mathrm{OH}]_{2} \mathrm{D}_{3}\right)$, may influence the apoptotic and necrotic cellular states of eosinophils. Blood eosinophils from atopic donors were incubated with physiologically relevant concentrations of extrarenal calcitriol (i.e., 0.05-100 nM) [22]. We observed a dose-dependent reduction of necrotic and apoptotic eosinophils along with an increase of viable eosinophils, in response to increasing doses of calcitriol after $24 \mathrm{~h}$ of incubation (Fig. 1).
The effect of calcitriol on eosinophil survival was significant when compared with media alone at a concentration of $10 \mathrm{nM}$. Therefore, $10 \mathrm{nM}$ of calcitriol was selected for future experiments ( $n=5$, IL-5 vs. calcitriol, $p<0.05$; Fig. 1b). So we demonstrated that calcitriol independently sustained the viability by reducing both apoptosis and necrosis in human blood-derived eosinophils for $24 \mathrm{~h}$ ex vivo, in the absence of an additional anti-apoptotic factor.

In order to evaluate the time course of calcitriol effects on eosinophil viability, we performed eosinophil survival assays using calcitriol (10 $\mathrm{nM}$ ) over 14 days. Using this time course, we observed that calcitriol on its own is insufficient to sustain eosinophil viability of $>75 \%$ over a long period, i.e., $>3$ days (Fig. 2). We consistently observed that media alone and calcitriol treatments showed reduced survival after day 4 of incubation, while IL-5 plus calcitriol promoted survival. After 3 days of incubation, there was no significant difference between the nontreated and calcitriol-treated eosinophils (day 3, vitamin D 34 $\pm 9 \%$ vs. media $16 \pm 9 \%, p>0.05, n=5$ ). However, when calcitriol was added to IL-5, we observe a marked increase of viable eosinophils on day 3 . We observed a significant potentiating effect of calcitriol when it was coincubated with IL-5 $(1 \mathrm{ng} / \mathrm{mL})$ after 4 days of culture. This effect not only persisted, but became elevated over the following 10 days. This difference between IL-5 plus calcitriol (10 nM) versus IL-5 alone occurred, respectively, at 7 days (20\%), 10 days (24\%) and 14 days (32\%). This finding depicts a synergistic effect of calcitriol with IL-5 beginning at day 7 (IL-5 + vitamin D $86 \pm 2 \%$ vs. IL-5 $66 \pm 2 \%$ and vitamin D $5 \pm 2 \%, p<0.05, n=12$; Fig. $2 \mathrm{a}$ ).

To determine if this coupled effect is specific to the IL-5 receptor, which signals through the IL-5, IL-3 and GM-CSF common $\beta$ chain [23], we tested if calcitriol could potentiate the prosurvival of another type of cytokine. Thus, IFN- $\gamma$ was chosen for a second viability assay. We monitored the effect of IFN- $\gamma(100 \mathrm{ng} / \mathrm{mL})$ in combination with calcitriol $(10 \mathrm{nM})$ on eosinophil survival over 7 days, as IFN- $\gamma$ is a less potent promoter of eosinophil survival than IL-5 [24]. Although IFN- $\gamma$ was, overall, less effective than IL-5 $(1 \mathrm{ng} / \mathrm{mL})$ in maintaining the viability of eosinophil populations, we were able to reproduce an analogous trend of effects in comparison to IL-5 and calcitriol experiments through the addition of both IFN- $\gamma$ $(100 \mathrm{ng} / \mathrm{mL})$ and calcitriol $(10 \mathrm{nM})$ treatment over the 7 -day period. Again, the difference in percentage of viable eosinophils between IFN- $\gamma$ and calcitriol versus IFN- $\gamma$ alone indicated a synergistic effect on viability at day 7 (IFN- $\gamma+$ vitamin D $44 \pm 9 \%$ vs. IFN- $\gamma 22 \pm 8 \%$ and vita$\min \mathrm{D} 7 \pm 2 \%, p<0.05, n=5$ ). 
Fig. 3. The number of eosinophilic cell particles is greatly reduced in the IL-5 (1 ng/ $\mathrm{mL})+$ calcitriol $(10 \mathrm{nM})$ treatment group. a Eosinophilic particles (left panel, left lower quadrant), are defined as low SS/low FS events using flow cytometry light-scattering properties. The number of cell particles corresponds to the percentage of necrotic eosinophils (right panel, upper right quadrant). b IL-5 + VD treatment reduced the relative percentage of cell particles in comparison to all other treatments $\left({ }^{* *} p<0.01\right.$, $n=10$ ). Eos/eos, eosinophils; FSC, forward scatter; SSC, side scatter; VD, vitamin D.

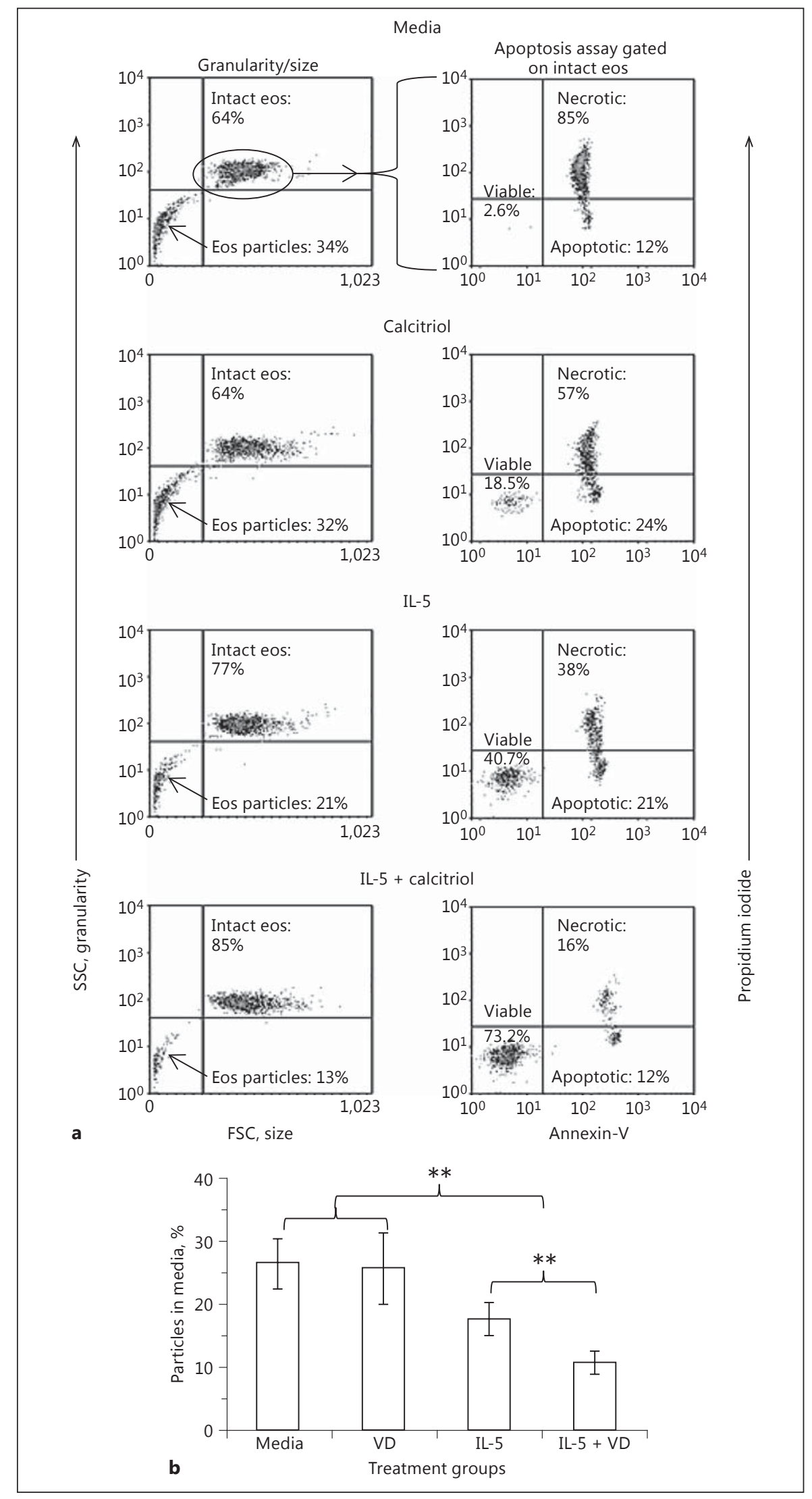

Calcitriol Reduces Eosinophil Release of Cytotoxic Granules
Int Arch Allergy Immunol 2016;171:119-129 DOI: $10.1159 / 000450951$ 
Fig. 4. Eosinophilic cell particles contain intact, membrane-bound CD63+/EPX+ granules. Eosinophilic cell particles from the nontreated group at day 7 of incubation contained a considerable number of granules (upper left panel, lower left quadrant), defined as double-positive events for CD63 and EPX (lower right panel, upper right quadrant) as well as free EPX, defined as single-positive events for EPX (lower right panel, upper left quadrant). Representative of 5 independent experiments. FACS plots were obtained by gating on low SS/low FS event population using light-scattering properties on flow cytometry. EPX, eosinophil peroxidase.
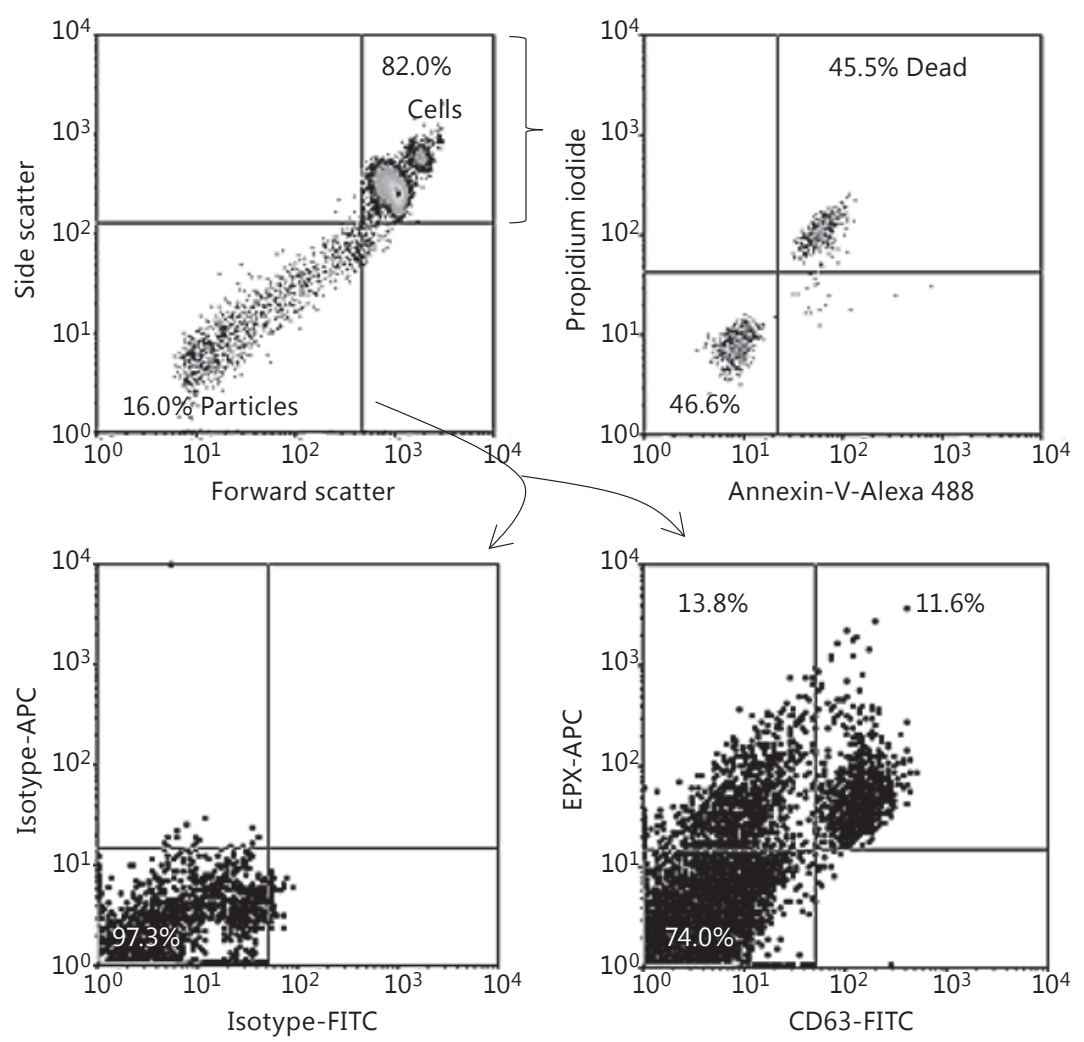

We thus confirmed that the effect of calcitriol on eosinophil survival is not solely dependent on IL-5 (Fig. 2b). Moreover, calcitriol does not appear to modulate the expression of the IL-5 receptor on eosinophils (online suppl. Fig. 1; see www.karger.com/doi/10.1159/000450951 for all online suppl. material). We further observed, despite the increase in eosinophil survival and complimentary decrease in necrosis, no significant increase in apoptosis (i.e., eosinophil annexin- $\mathrm{V}$ single-positives) throughout the long time course for both combination treatments, i.e., IL-5 + vitamin D and IFN- $\gamma+$ vitamin $\mathrm{D}$ (data not shown).

\section{Calcitriol Influences Necrotic Release}

We then explored the idea whether the observed effects of calcitriol on increasing eosinophil viability correlate with the inhibition of eosinophil necrosis, which, consequently, would reduce the release of intact, membranebound granules and proinflammatory mediators into the media, caused by this cytolytic process. This avenue was investigated due to observations that increased small par- ticles appeared in the media, which, it was assumed, resulted from eosinophil necrotic death; on flow cytometry, these particles can, based on their low forward scatter/side scatter (FS/SS) properties, be distinguished from intact cells. As demonstrated in Figure 3a, the number of particles appears to correlate with an increased incidence of necrosis in cells cultured in media alone (right panel, upper right quadrant). Treatment with IL-5 and calcitriol for a duration of 7 days resulted in increased high FS/SS events (left panel, upper right quadrant), corresponding to intact eosinophils, versus low FS/SS events (left panel, lower left quadrant), corresponding to particles. The most significant decrease in particles occurred within the IL-5 $(1 \mathrm{ng} / \mathrm{mL})$ plus calcitriol $(10 \mathrm{nM})$ treatment population (Fig. 3a). The percentage of particles in media resulting from the coincubation of IL-5 and calcitriol was significantly lower than treatment with IL- 5 only (IL- $5+$ vitamin D $10.8 \pm 1.8 \%$ vs. IL-5 $17.7 \pm 2.6 \%, p=<0.05, n=10$; Fig. 3b). We observed that the IL-5 and calcitriol combination decreased the number of particles in a pattern that correlated with the level of eosinophil necrosis (Fig. 3a, b). 


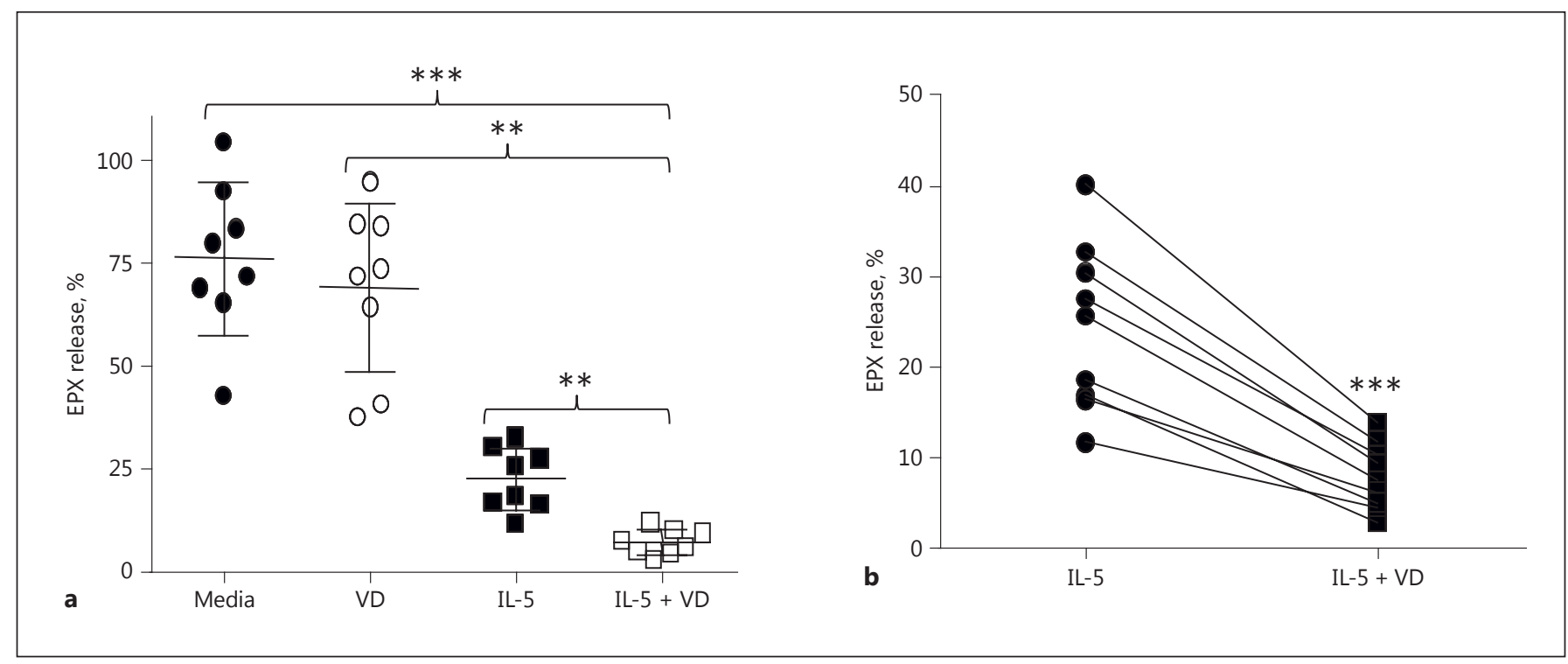

Fig. 5. Calcitriol, when combined with IL-5, minimizes the spontaneous release of active EPX in culture media. a All treatment groups had significantly less active EPX released in comparison to the IL-5 + VD group (ANOVA; ${ }^{* *} p<0.01,{ }^{* * *} p<0.001, n=9$ ). b The relationship between IL- 5 and IL-5 + VD treatments consis- tently depicts a respective decrease in EPX percentage within each experiment in a significant manner (paired $t$ test; ${ }^{* * *} p<0.001$, $n=9$ ). EPX activity in the media was measured using an OPDbased colorimetric assay. EPX, eosinophil peroxidase; VD, vita$\min \mathrm{D}$.
Since the nature of particles is complex, we wished to determine the presence of intact granules in the extracellular media as well as the cytotoxic mediators stored in the eosinophil granules. Therefore, we stained permeabilized and nonpermeabilized eosinophil preparations following a 7-day treatment for granules in media only, using the CD63 granule surface marker and EPX, a cytotoxic protein unique to eosinophil granules. We found that membrane-bound CD63+/EPX+ intact granules were indeed present in the particle populations from eosinophil preparations in media alone after 7 days (Fig. 4).

To investigate the spontaneous release of active EPX from eosinophils, we measured the level of active EPX in culture media following treatment. These results revealed a lower abundance of active EPX in the media supernatant containing calcitriol (10 nM) in comparison with their respective controls (Fig. 5a). Moreover, all other treatment groups had significantly more EPX release than the IL-5 plus vitamin D group (IL-5 + vitamin D $8 \pm 1 \%$ vs. IL-5 $24 \pm 3 \%$ vs. vitamin D $64 \pm 8 \%$ vs. media $72 \pm 7 \%, p<0.01, n=9$ ). We observed a consistent decrease in EPX release by adding calcitriol $(10 \mathrm{nM})$ directly and compared to IL-5 (1 ng/mL) alone (Fig. 5b). These results depict a tendency for EPX release during eosinophil cytolysis which is analogous to the trends in granule release (and necrosis), yet complementary to viability patterns.

We next determined if calcitriol would impact eosinophil sensitivity to a secretagogue such as PAF. We found that calcitriol treatment alone did not affect viable eosinophil EPX release in response to PAF at day 7 (Fig. 6). In fact, only the treatment groups containing IL-5 $(1 \mathrm{ng} / \mathrm{mL})$ were capable of releasing substantial EPX upon PAF stimulation, while other groups lacking IL-5 were less actively degranulating (Fig. 6). In addition, the IL-5 and IL-5 + vitamin $\mathrm{D}$ groups were not significantly different from one another. Hence, media and vitamin D data were similar while IL-5 and IL-5 + vitamin D results were elevated similarly. Overall, these results show that the effect of calcitriol on EPX release obtained by PAF stimulation had an inverse relationship to the effect of spontaneous EPX discharge. Again, the sensitivity to PAF seems to reflect levels of viable eosinophils in all treatment groups at day 7 (Fig. 2a, 5a). By matching data from the various EPX assays to their respective viability data, we observed a significant inverse correlation between the amount of EPX observed in the media and eosinophil viability $\left(r^{2}=\right.$ 0.9854, $p<0.05$; Fig. 7). 


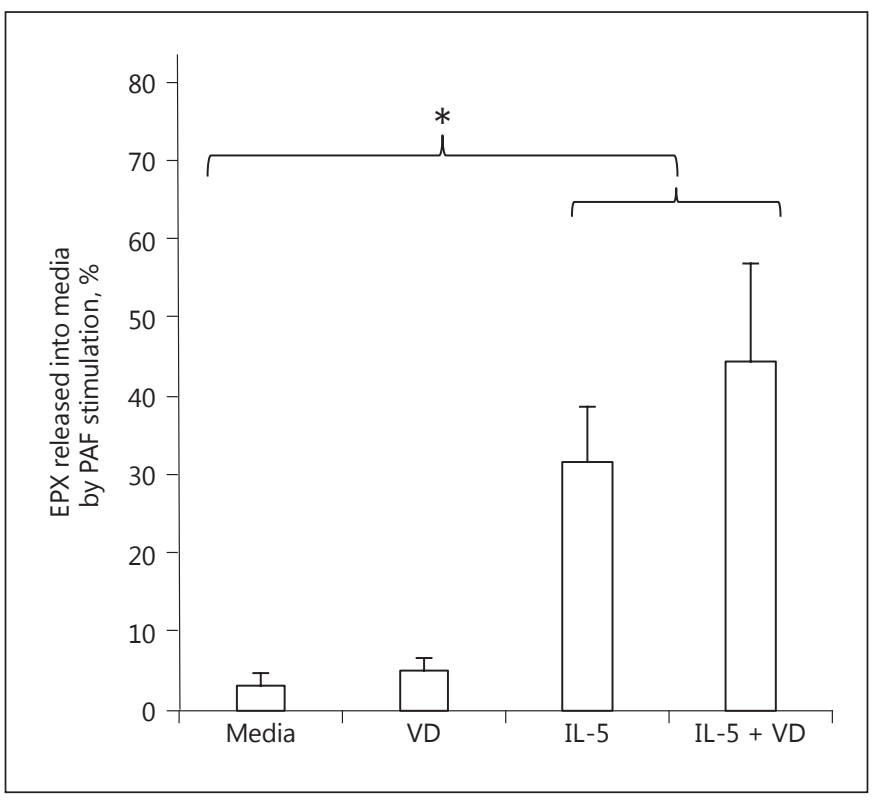

Fig. 6. The addition of calcitriol to IL-5-treated eosinophils maintains eosinophil sensitivity to PAF ex vivo. Treatment with IL-5 and IL-5 + VD caused significantly higher PAF-induced EPX release than media + VD (day 7 * $^{*} p<0.05 ; n=9$ ). IL-5 and IL-5 + VD were not significantly different. EPX activity in media was measured using an OPD-based colorimetric assay. EPX, eosinophil peroxidase; PAF, platelet-activating factor; VD, vitamin D.

\section{Discussion}

This is the first study to demonstrate an effect by vitamin D on eosinophil survival and cytolysis. Relating to eosinophil survival, we first confirmed a previous report showing that calcitriol (100 nM) sustained eosinophil viability for up to $72 \mathrm{~h}$ [20]. However, we have extended this finding by showing a similar effect with a 10 -fold-lower concentration of calcitriol (10 nM). Both concentrations of calcitriol are viable in the lung mucosal microenvironment, since the lung epithelium, containing 1a-hydroxylase, has been reported to convert calcifediol into calcitriol at much greater levels in vitro. Hansdottir et al. [25] observed that approximately 1,000 $\mathrm{nM}$ of calcifediol was transformed in a constitutive manner into $0.6 \mathrm{nM}$ of calcitriol after $24 \mathrm{~h}$, allowing a high calcitriol concentration at the mucosal level. This conversion can also be done by alveolar macrophages, dendritic cells and lymphocytes, implying that the extrarenal expression of $1 \alpha$-hydroxylase is specific to the immune system $[22,26]$.

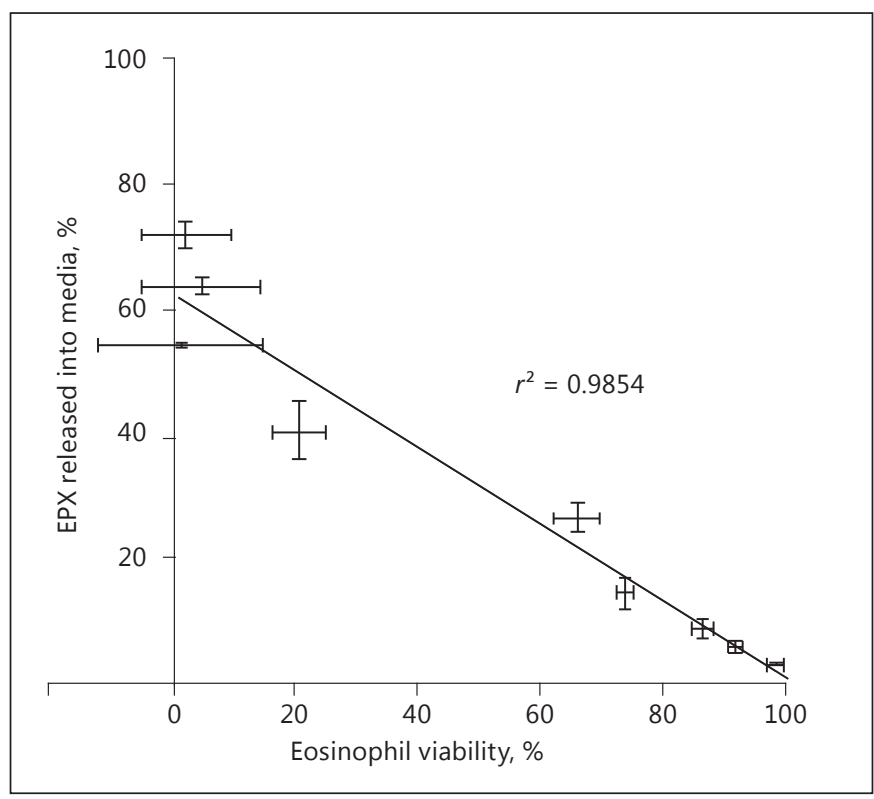

Fig. 7. EPX activity in media is inversely correlated to eosinophil survival. The percentage of EPX release into media and levels of eosinophil survival are inversely correlated with high significance $\left(r^{2}=0.9854, p<0.05, n=4-11\right)$. Results from EPX colorimetric assays were paired with corresponding eosinophil viability data from annexin-V/PI assays. EPX activity in media was measured by OPD-based colorimetric assay. Viability was determined by annexin-V binding and PI exclusion by flow cytometry. EPX, eosinophil peroxidase.

In addition, we showed a synergistic effect by combining calcitriol with antiapoptotic cytokines (i.e., IL-5 and IFN- $\gamma$ ). We tested IFN- $\gamma$ in order to demonstrate that the potentiating effect of calcitriol on eosinophil survival was not exclusive to IL-5 receptor signaling. IFN- $\gamma$ was chosen due to its signaling initiation, which relies on IFNGR1 and 2 (which interact with JAK1 and 2, respectively), being independent from IL-5 receptor $\beta$ chain signaling (which relies on $\beta$ receptor activation to phosphorylate JAK2 exclusively) [23]. The synergism observed when calcitriol and the tested cytokines were combined is indicative that calcitriol triggers a cell survival/death pathway that is distinct from apoptosis. In combination with the lack of changes in apoptotic eosinophil levels and production of cell particles at this same time point, we propose a necrotic pathway as a target for intracellular modulation by calcitriol on eosinophils. In general, our results were in contrast to our original notion that calcitriol may decrease eosinophil survival, similar to its effect on cancer cells. 
Eosinophils are usually known to release their granules by exocytosis, which can occur through the regulated process of piecemeal degranulation $[27,28]$. The discharge of eosinophil granules can also be the result of cytolysis (related to necrotic death). Necrosis is often defined as a proinflammatory event, since the liberation of intracellular contents into the tissues from eosinophils allows the contents to act as natural adjuvants which can initiate various immunological activation mechanisms [29]. This is in contrast to apoptosis, which is a prominent anti-inflammatory mechanism for controlling immune cell populations. In asthmatics, apoptotic eosinophils can be detected in the sputum during recovery from acute asthma exacerbations that require corticosteroid treatment. This suggests that eosinophil apoptosis is important in the clearance of airway eosinophils for the resolution of inflammatory responses [30]. Moreover, apoptotic eosinophils are readily engulfed by macrophages, which can be detected in the sputum or airways [30]. Hence, promoting the apoptotic pathway, in preference to necrosis, is one of the current therapeutic approaches for asthma management $[1,31]$. Interestingly, there are observations of bronchial tissue that seem to lack apoptotic eosinophils. Research by Kodama et al. [32] failed to show apoptotic eosinophils in mouse tissues following allergic inflammation. Moreover, evidence suggests that cytolytic eosinophils and eosinophilic granules are more commonly found in the allergic airway mucosa and in eosinophil esophagitis $[6,16]$. In humans, allergen exposure causes mucosal eosinophilia, accompanied by eosinophil piecemeal degranulation, eosinophil cytolysis, and the deposition of granules [14]. The presence of cell-free granules in the bronchial mucosa also correlates with asthma severity $[7,33,34]$. In fact, epithelial damage and loss as a result of the specific eosinophil granule proteins, major basic protein, eosinophil cationic protein, and EPX, are collectively believed to play a significant role in asthma pathology. It is proposed that major basic protein and EPX are directly involved in epithelial damage, bronchospasm, and increasing vascular permeability in the lung [35].

Calcitriol was able to significantly reduce the spontaneous release of EPX by eosinophils but not PAF-induced EPX release, when combined with an antiapoptotic cytokine. PAF induces many eosinophil functions in inflammatory responses, so it may be an important factor for eosinophil degranulation in vivo [36]. Examining eosinophil responses to PAF offers insight into the proinflammatory capacity of eosinophils via cytotoxic mediator release upon stimulation. Furthermore, investigating the

Calcitriol Reduces Eosinophil Release of

Cytotoxic Granules effect of calcitriol in relation to PAF sensitivity may elucidate vitamin $\mathrm{D}$ anti-inflammatory mechanisms. In our study, we showed that calcitriol did not affect eosinophil PAF-sensitivity. This finding was unexpected, since calcitriol suppressed spontaneous EPX release. Interestingly, eosinophil PAF-induced degranulation of EPX followed a trend towards a pattern of viability at day 7 (Fig. 2a). Groups marked by more abundant viable eosinophils (i.e., IL-5 and IL-5 + vitamin D) maintained PAF sensitivity, indicating that PAF responsiveness was likely related to a higher number of viable cells. This parallel finding confirms that viable eosinophils, whether exposed to calcitriol or not, are able to release cytotoxic mediators after 7 days of incubation. We thus conclude that calcitriol does not selectively modulate stimulated eosinophil degranulation responses to PAF.

More than 1,000 genes are under the control of the vitamin $\mathrm{D}$ receptor, which is essential for directly mediating the various effects of vitamin D [37]. Accordingly, vitamin $\mathrm{D}$ deficiency in humans has been associated with an increase (and/or adverse outcomes) in autoimmune diseases, infections, cardiovascular diseases, and cancer, and is apparently linked to a higher risk of all-cause mortality [19]. Direct evidence supporting a role for vitamin $\mathrm{D}$ in asthma has been revealed from genetic studies of vitamin $\mathrm{D}$ receptor polymorphisms as a risk factor in different human populations [38-41]. Vitamin D deficiency has also been studied in relation to the emergence of airway diseases, along with the hypothesis that a deficiency of vitamin $\mathrm{D}$ is associated with immune system impairment, leading to inflammatory and autoimmune manifestations. In multiple studies, low serum vitamin D (25$\left.[\mathrm{OH}] \mathrm{D}_{3}\right)$ has been associated with poor lung function as well as increased airway hyperactivity, allergy, and asthma, in adults as well as in adolescents and children [42-44]. Lung epithelial cells express the enzyme $1 a$-hydroxylase, which catalyzes the conversion of the vitamin $\mathrm{D}$ precursor calcidiol $\left(25-[\mathrm{OH}] \mathrm{D}_{3}\right)$ to the active form, calcitriol $\left(1 \alpha, 25-[\mathrm{OH}]_{2} \mathrm{D}_{3}\right)$ [25]. This function of the lung epithelia suggests that local concentrations of calcitriol are able to rise independently from circulating levels, and may reach higher concentrations than systemic vitamin D. It also implies an immunologic role for this particular microenvironment, beyond calcium regulation.

We also determined the presence of eosinophil granules in particles present in the media, which suggests that this could be a useful indicator of eosinophil necrosis. Besides confirming the presence of free EPX, the dispersion of $\mathrm{CD} 63+/ \mathrm{EPX}+$ granules within particles in the media is 
suggestive of direct intact granule release. The association between particle levels and necrotic cell counts in our study is in line with the documented phenomenon of cellfree granules in eosinophilic disease [7]. Cytolysis, as cell demise, causes the discharge of diverse cellular components, including intact membrane-bound granules, into the surrounding environment. Indeed, whole, extracellular eosinophil granules have been reported to release their content upon receptor stimulation [45]. In allergic asthma, sustaining eosinophil viability with calcitriol may at first appear an unfavorable outcome, but the consecutive reduction of granule deposition might result in less mucosal damage and inflammation, supporting e notion of an anti-inflammatory role for calcitriol in this context. In conclusion, our data suggest a new antinecrotic/antiapoptotic function for calcitriol, and possibly other vitamin D metabolites that will need to be studied further.

\section{Acknowledgements}

This work was supported by the Vitamin Fund/ALES/University of Alberta.

\section{Authors' Contribution}

C.E. was primarily responsible for the acquisition, analysis, and interpretation of data. C.E. and F.D. wrote the paper. P.L. also contributed to the design of the study, data analysis and critical review of the manuscript. Y.W. contributed to the optimization of the EPX assay and the collection of data. L.C. provided important intellectual review of the scientific content. L.C. and P.L. supervised C.E., with F.D. being the principal investigator and supervisor for this study, from its conception (design, analysis, and interpretation) to writing the paper and final approval.

\section{Disclosure Statement}

There are no conflicts of interest to disclose.

\section{References}

1 Wegmann M: Targeting eosinophil biology in asthma therapy. Am J Respir Cell Mol Biol 2011;45:667-674.

2 Fulkerson PC, Rothenberg ME: Targeting eosinophils in allergy, inflammation and beyond. Nat Rev Drug Discov 2013;12:117-129.

3 Martin LB, Kita H, Leiferman KM, Gleich GJ: Eosinophils in allergy: role in disease, degranulation, and cytokines. Int Arch Allergy Immunol 1996;109:207-215.

4 Kroegel C, Warner JA, Virchow JC Jr, Matthys H: Pulmonary immune cells in health and disease: the eosinophil leucocyte (part II). Eur Respir J 1994;7:743-760.

5 Kita H: Eosinophils: multifaceted biological properties and roles in health and disease. Immunol Rev 2011;242:161-177.

6 Erjefalt JS, Persson CG: New aspects of degranulation and fates of airway mucosal eosinophils. Am J Respir Crit Care Med 2000; 161:2074-2085.

7 Persson CG, Erjefalt JS: Eosinophil lysis and free granules: an in vivo paradigm for cell activation and drug development. Trends Pharmacol Sci 1997;18:117-123.

8 Tumes DJ, Cormie J, Calvert MG, Stewart K, Nassenstein C, Braun A, et al: Strain-dependent resistance to allergen-induced lung pathophysiology in mice correlates with rate of apoptosis of lung-derived eosinophils. J Leukoc Biol 2007;81:1362-1373.

9 Ohnmacht C, Pullner A, van Rooijen N, Voehringer D: Analysis of eosinophil turnover in vivo reveals their active recruitment to and prolonged survival in the peritoneal cavity. J Immunol 2007;179:4766-4774.
10 Kankaanranta H, Moilanen E, Zhang X: Pharmacological regulation of human eosinophil apoptosis. Curr Drug Targets Inflamm Allergy 2005;4:433-445.

11 Simon HU, Yousefi S, Schranz C, Schapowal A, Bachert C, Blaser K: Direct demonstration of delayed eosinophil apoptosis as a mechanism causing tissue eosinophilia. J Immunol 1997; 158:3902-3908.

12 Simon HU: Molecular mechanisms of defective eosinophil apoptosis in diseases associated with eosinophilia. Int Arch Allergy Immunol 1997;113:206-208.

13 Erjefalt JS, Sundler F, Persson CG: Eosinophils, neutrophils, and venular gaps in the airway mucosa at epithelial removal-restitution. Am J Respir Crit Care Med 1996;153:16661674.

14 Erjefalt JS, Andersson M, Greiff L, Korsgren M, Gizycki M, Jeffery PK, et al: Cytolysis and piecemeal degranulation as distinct modes of activation of airway mucosal eosinophils. J Allergy Clin Immunol 1998;102:286-294.

15 Greiff L, Erjefalt JS, Andersson M, Svensson C, Persson CG: Generation of clusters of free eosinophil granules (Cfegs) in seasonal allergic rhinitis. Allergy 1998;53:200-203.

16 Saffari H, Hoffman LH, Peterson KA, Fang JC, Leiferman KM, Pease LF 3rd, et al: Electron microscopy elucidates eosinophil degranulation patterns in patients with eosinophilic esophagitis. J Allergy Clin Immunol 2014;133:1728-1734.e1.

17 Rothenberg ME: Eosinophilia. N Engl J Med 1998;338:1592-1600
18 Picotto G, Liaudat AC, Bohl L, Tolosa de Talamoni N: Molecular aspects of vitamin D anticancer activity. Cancer Invest 2012;30:604614

19 Holick MF: Vitamin D deficiency. N Engl J Med 2007;357:266-281.

20 Hiraguchi Y, Tanida H, Sugimoto M, Hosoki $K$, Nagao M, Tokuda R, et al: 1,25-Dihydroxyvitamin D3 upregulates functional C-XC chemokine receptor type 4 expression in human eosinophils. Int Arch Allergy Immunol 2012;158(suppl 1):51-57.

21 Davoine F, Cao M, Wu Y, Ajamian F, Ilarraza $\mathrm{R}$, Kokaji AI, et al: Virus-induced eosinophil mediator release requires antigen-presenting and CD4+ T cells. J Allergy Clin Immunol 2008;122:69-77, e1-e2.

22 Hewison M, Adams JS: Extrarenal 1 $\alpha$-hydroxylase; in: Adams JS, Feldman D, Wesley Pike J (eds): Vitamin D, ed 3, chapt 45. San Diego, Academic Press, 2011, pp $777-$ 804.

23 Hercus TR, Dhagat U, Kan WL, Broughton SE, Nero TL, Perugini M, et al: Signalling by the $\beta \mathrm{c}$ family of cytokines. Cytokine Growth Factor Rev 2013;24:189-201.

24 Valerius T, Repp R, Kalden JR, Platzer E: Effects of IFN on human eosinophils in comparison with other cytokines. A novel class of eosinophil activators with delayed onset of action. J Immunol 1990;145:2950-2958.

25 Hansdottir S, Monick MM, Hinde SL, Lovan N, Look DC, Hunninghake GW: Respiratory epithelial cells convert inactive vitamin $\mathrm{D}$ to its active form: potential effects on host defense. J Immunol 2008;181:7090-7099. 
26 Hansdottir S, Monick MM: Vitamin D effects on lung immunity and respiratory diseases. Vitam Horm 2011;86:217-237.

27 Melo RC, Dvorak AM, Weller PF: Contributions of electron microscopy to understand secretion of immune mediators by human eosinophils. Microsc Microanal 2010;16:653660.

28 Kim JD, Willetts L, Ochkur S, Srivastava N, Hamburg R, Shayeganpour A, et al: An essential role for Rab27a GTPase in eosinophil exocytosis. J Leukoc Biol 2013;94:1265-1274.

29 Han J, Zhong CQ, Zhang DW: Programmed necrosis: backup to and competitor with apoptosis in the immune system. Nat Immunol 2011;12:1143-1149.

30 Woolley KL, Gibson PG, Carty K, Wilson AJ, Twaddell SH, Woolley MJ: Eosinophil apoptosis and the resolution of airway inflammation in asthma. Am J Respir Crit Care Med 1996;154:237-243.

31 Hallett JM, Leitch AE, Riley NA, Duffin R, Haslett C, Rossi AG: Novel pharmacological strategies for driving inflammatory cell apoptosis and enhancing the resolution of inflammation. Trends Pharmacol Sci 2008;29: 250-257.

32 Kodama T, Matsuyama T, Miyata S, Nishimura H, Nishioka Y, Kitada O, et al: Kinetics of apoptosis in the lung of mice with allergic airway inflammation. Clin Exp Allergy 1998;28: 1435-1443.
33 Frigas E, Loegering DA, Solley GO, Farrow GM, Gleich GJ: Elevated levels of the eosinophil granule major basic protein in the sputum of patients with bronchial asthma. Mayo Clin Proc 1981;56:345-353.

34 Filley WV, Holley KE, Kephart GM, Gleich GJ: Identification by immunofluorescence of eosinophil granule major basic protein in lung tissues of patients with bronchial asthma. Lancet 1982;2:11-16.

35 Gleich GJ: Mechanisms of eosinophil-associated inflammation. J Allergy Clin Immunol 2000; 105:651-663.

36 Dyer KD, Percopo CM, Xie Z, Yang Z, Kim JD, Davoine F, et al: Mouse and human eosinophils degranulate in response to plateletactivating factor (PAF) and lysoPAF via a PAF-receptor-independent mechanism: evidence for a novel receptor. J Immunol 2010; 184:6327-6334.

37 Tavera-Mendoza LE, White JH: Cell defenses and the sunshine vitamin. Sci Am 2007;297: $62-65,68-70,72$

38 Saadi A, Gao G, Li H, Wei C, Gong Y, Liu Q: Association study between vitamin $\mathrm{D}$ receptor gene polymorphisms and asthma in the Chinese Han population: a case-control study. BMC Med Genet 2009;10:71.
39 Bosse Y, Lemire M, Poon AH, Daley D, He JQ, Sandford A, et al: Asthma and genes encoding components of the vitamin $D$ pathway. Respir Res 2009;10:98

40 Bosse Y, Maghni K, Hudson TJ: 1alpha,25dihydroxy-vitamin D3 stimulation of bronchial smooth muscle cells induces autocrine, contractility, and remodeling processes. Physiol Genomics 2007;29:161-168.

41 Wjst M: Variants in the vitamin D receptor gene and asthma. BMC Genet 2005;6:2.

42 Sandhu MS, Casale TB: The role of vitamin D in asthma. Ann Allergy Asthma Immunol 2010;105:191-199.

43 Dogru M, Kirmizibekmez H, Yesiltepe Mutlu RG, Aktas A, Ozturkmen S: Clinical effects of vitamin D in children with asthma. Int Arch Allergy Immunol 2014;164:319-325.

44 Di Filippo P, Scaparrotta A, Rapino D, Cingolani A, Attanasi M, Petrosino MI, et al: Vitamin D supplementation modulates the immune system and improves atopic dermatitis in children. Int Arch Allergy Immunol 2015; 166:91-96.

45 Neves JS, Perez SA, Spencer LA, Melo RC, Reynolds L, Ghiran I, et al: Eosinophil granules function extracellularly as receptor-mediated secretory organelles. Proc Natl Acad Sci USA 2008; 105:18478-18483. 